\title{
VACStent: Combining the benefits of endoscopic vacuum therapy and covered stents for upper gastrointestinal tract leakage
}

다)(앙

\author{
Authors \\ Institutions \\ 1 Department of Abdominal, Tumor, Transplant and \\ Vascular Surgery, Cologne-Merheim Medical Center, \\ University Witten/Herdecke, Cologne, Germany \\ 2 Department of Gastroenterology, Cologne-Holweide \\ and Merheim Medical Center, Cologne, Germany
}

Jonas Lange ${ }^{1}$, Arno Dormann², Dirk Rolf Bulian¹, Ulrich Hügle², Claus Ferdinand Eisenberger ${ }^{1}$, Markus Maria Heiss submitted 19.11 .2020

accepted after revision 22.2 .2021

\section{Bibliography}

Endosc Int Open 2021; 09: E971-E976

DOI 10.1055/a-1474-9932

ISSN 2364-3722

(C) 2021. The Author(s).

This is an open access article published by Thieme under the terms of the Creative Commons Attribution-NonDerivative-NonCommercial License, permitting copying and reproduction so long as the original work is given appropriate credit. Contents may not be used for commercial purposes, or adapted, remixed, transformed or built upon. (https://creativecommons.org/licenses/by-nc-nd/4.0/)

Georg Thieme Verlag KG, Rüdigerstraße 14,

70469 Stuttgart, Germany

Corresponding author

M.M. Heiss, MD, FACS, Department of Abdominal, Tumor, Transplant and Vascular Surgery, Cologne-Merheim Medical Center, Witten/Herdecke University, Ostmerheimer Strasse 200, D-51109 Cologne, Germany

Fax: 0049-221-8907-8561

heissm@kliniken-koeln.de

\section{ABSTRACT}

Background and study aims Endoscopic treatment has markedly improved the high morbidity and mortality in patients with upper gastrointestinal tract leakage. Most pro- cedures employ either covered self-expanding metal stents (SEMS) or endoscopic vacuum therapy (EVT), both with good clinical success but also with concomitant significant shortcomings inherent in each technique. A newly developed device, the VACStent, combines the fully covered SEMS with a polyurethane sponge cylinder anchored on the outside. This allows endoluminal EVT while keeping the intestinal lumen patent. The benefit is prevention of stent migration because the suction force of the sponge-cylinder immobilizes the VACStent on the intestinal wall, while at the same time, the attached external vacuum pump suctions off any secretions and improves healing with negative-pressure wound treatment (NPWT).

Patients and methods In this pilot study, the first patients to receive the VACStent were assessed. Outcomes included the applicability and stability of the VACStent system together with the clinical course.

Results Three patients with different clinical courses were managed with the VACStent. The first patient suffered anastomotic leakage following subtotal esophagectomy and was successfully treated with two postoperative VACStents for 12 days. The second patient received a covered SEMS for 14 days for acute Boerhaave syndrome. Due to persistent leakage, management was converted to EVT. Seven days, later a VACStent was inserted to allow oral nutrition while the leak finally closed. In the third patient, a LINX Reflux Management System had to be removed for erosion, leaving the gastroesophageal junction (GEJ) with a full-thickness gap. After VACstent insertion, successful closure was achieved within 4 days.

Conclusions These clinical cases demonstrate the applicability and efficacy of the VACstent in management of esophageal and anastomotic leakage. With its vacuum sponge, the stent fosters wound healing while the covered SEMS keeps the passage patent for nutrition.

\section{Introduction}

Clinical management of upper gastrointestinal tract leakage with septic sequelae has markedly improved since the introduction of endoluminal sealing techniques. This has mainly been accomplished either with covered stents or endoscopic vacuum therapy [1-3]. However, both techniques are hampered by inherent shortcomings. SEMS are burdened by a migration rate of $50 \%$ or more, and because they exert a radial force on the intestinal wall, they impair microperfusion and wound healing 
at the leakage site [4,5]. Endoluminal vacuum therapy improves wound healing and drains inflammatory secretions via the suction-induced negative pressure $[6,7]$. The latter, on the other hand, induces positive back-pressure of the same magnitude in the opposite direction (Newton's third law of action and reaction), which puts a strain on the intestinal wall and occludes the lumen. This then might induce continuous microaspiration. Upper gastrointestinal endoluminal EVT therefore mandates additional feeding-tube running alongside or through the sponge.

The new VACStent concept combines the benefits of both techniques, sealing the intestinal wound with a negative-pressure sponge and ensuring patent intestinal passage for fluids and nutrition. This is accomplished by a fully covered SEMS with a sponge-cylinder affixed to its exterior, which in turn is connected by catheter tubing to a vacuum pump. Three initial clinical cases are presented here as examples for the proposed application of this new endoscopic device.

\section{Patients and methods}

The VACStent comprises a self-expanding nitinol stent covered with a silicone-membrane impermeable to liquid and gas (VacStent Medtech AG, Steinhausen, Switzerland). Affixed to the exterior of the stent is a polyurethane sponge cylinder (thickness $10 \mathrm{~mm}$ ) connected to an external vacuum pump via a fixed small gauge (12 F) catheter ( $\mathbf{F i g . 1 a , ~}>$ Fig. 1b). There are multiple holes at the distal end of the catheter over the length of the sponge cylinder. The lumen of the $50 \mathrm{~mm}$ long sponge cylinder has an inside diameter of $12 \mathrm{~mm}$ and is framed by the flanged ends of the $30-\mathrm{mm}$ diameter stent. Once negative pressure is applied, only the flanged ends of the stent are in contact with the intestinal wall, sealing the sponge cylinder against the intestinal fluids. This constant suction induces a force of the same magnitude - but in opposite direction - as the applied negative pressure, thereby immobilizing the VACStent on the intestinal wall and preventing stent-migration. The VACStent is loaded on a flexible introducer system (12-mm diameter), which is inserted transorally in over-the-wire technique. Positioning and release is monitored either by fluoroscopy or more simply through a small endoscope paralleling the inserted introducer system. If the positioning is controlled by fluoroscopy, the unfolding of the stent can easily be followed on screen. If controlled by direct vision using a small endoscope, leak position is determined by using the measured distance from the leak to the front teeth and visualizing proximal deployment of the sponge.

After release, the nitinol filaments unfold the VACStent to its original shape ( $\mathbf{F i g . 1 C}$ ). Because of the open-pored sponge, a vacuum is created all over the contact plane between the sponge cylinder and the esophageal wall. This means that the suction functions independently from the position of the catheter. There are markers at the ends of the VACStent that facilitate orientation, if applied fluoroscopy.

If the VACStent is deployed too distally, it is easy to reposition the device more proximally by grasping the circular wire mounted at the proximal bulged end with a forceps and just
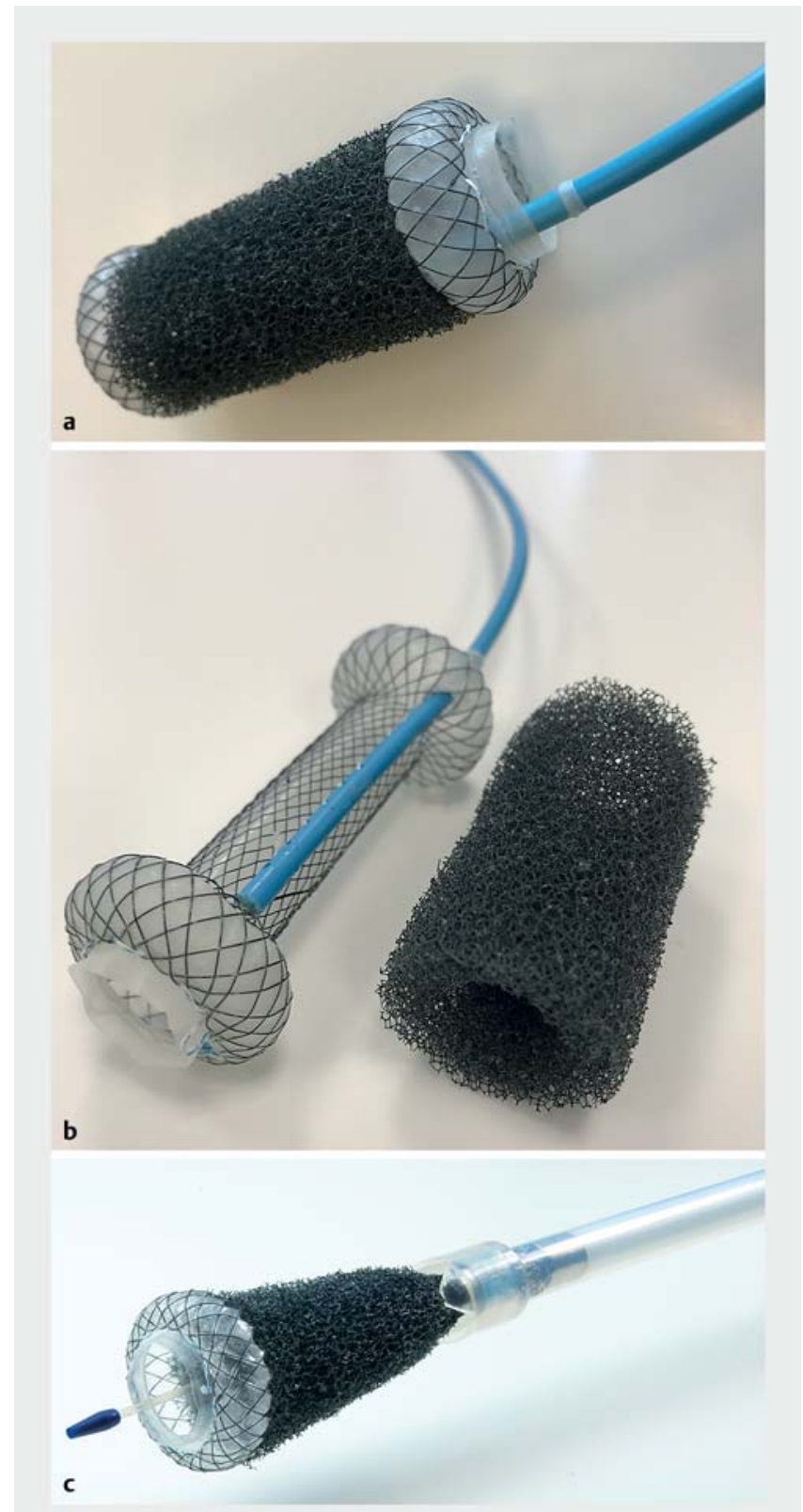

- Fig. 1 a VAC Stent: a silicone-coated Nitiolstent covered by a PU sponge cylinder at the outside connected by a tube to the vacuum pump. b Components of the VAC Stent: a silicone-coated Nitiolstent, a Polyurethan Sponge cylinder and a suction tube connected to vacuum pump. c After release the nitinol filaments unfold the VACStent to its original shape.

pull, because this reduces the stent diameter. The same procedure is used to extract the device. The repositioned transnasal suction catheter is connected to a vacuum pump. The applied negative pressure ranges from $-80 \mathrm{~mm} \mathrm{Hg}$ to $-120 \mathrm{mmHg}$. However, continuous suction of secretions and VACStent fixation on the intestinal wall is also possible with vacuum pressure down to $-50 \mathrm{mmHg}[3,8]$.

This study was a retrospective analysis of prospectively collected data from patients selected for an applicability trial of the new VACStent system [9]. The study protocol was approved 
of the Institutional Review Board of the University Witten/Herdecke. Three patients were selected to study in whom the postulated benefits of this hybrid sponge-stent system were realized in clinical practice. While the main focus was on the applicability and migration resistance of the VACStent, the study also investigated sealing of different leak configurations and unrestrained passage of swallowed liquids through the stent.

\section{Results}

The first patient had undergone subtotal esophagectomy with esophago-jejunal circular stapler anastomosis for adenocarcinoma. On postoperative day 14, endoscopy confirmed anastomotic leakage with a small abscess cavity ( $\mathbf{F i g} \cdot \mathbf{2 a}$ ). A VACStent was inserted, which sealed off the leak as demonstrated by contrast fluoroscopy. Suction with a continuous negative pressure of $-120 \mathrm{mmHg}$ was instituted. Control thoracic computed tomography on day 4 showed the exact VACStent position with no mediastinal collection ( $\mathbf{F i g . 2 b}$ ). On day 5 , the VACStent was removed and the area treated was examined. Good induction of granulation tissue was seen, indicating active wound healing ( $\mathbf{F i g} \cdot \mathbf{2 c}$ ). A second VACStent was inserted and the negative pressure lowered to $-80 \mathrm{mmHg}$. After another 7 days of continuous suction, the VACStent was removed ( $\triangleright$ Fig.2d). The leakage site was clean and only viable granulation tissue was evident, leaving a small cavity of 3 to $4 \mathrm{~mm}$ in depth, which was completely sealed off as demonstrated by contrast X-ray. Follow-up endoscopy 2 and 4 weeks later showed no evidence of stenosis.

The second patient, a 66-year-old woman with acute Boerhaave syndrome (esophageal tear from $29 \mathrm{~cm}$ to $36 \mathrm{~cm}$ ), was treated with a covered SEMS. She demonstrated signs of sepsis, most probably due to mediastinitis, but without a distinct fluid collection suitable for percutaneous drainage. In addition, a tube was inserted into her right thorax. After 14 days the SEMS was retrieved, and because of a stil- persistent esophageal tear from 30 to $34 \mathrm{~cm}$, management was converted to EVT (Esosponge, BBraun). The patient improved clinically and 7 days later the sponge was retrieved. However, the still-persistent leak re-

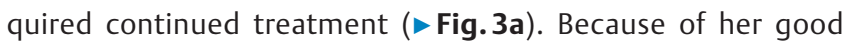
physical and neurological status with no need for a ventilator, a VACStent was inserted to allow oral nutrition ( $\mathbf{F i g} \mathbf{3 b}$ ). This was monitored fluoroscopically by upper gastrointestinal tract contrast series with the patient fully conscious ( $\mathbf{F i g . 3 c}$ ). This study also confirmed that the esophageal tear was fully sealed off ( $\triangleright$ Fig. 3d). At VACStent removal after 5 days, the leak was found to be closed by extensive granulation tissue, leaving no residual cavity ( $>$ Fig.3e). This was monitored during 6 -month follow-up, during which, the patient had no residual clinical symptoms ( $>$ Fig. $\mathbf{3 f}$ ).

The third patient was a 75-year-old woman treated for severe dysphagia due to a stenosis induced by a migrated magnetic LINX band (Johnson \& Johnson, United States), which had been implanted 2.5 years previously. Gastroscopy demonstrated endoluminal invasion of the metal band over one-third of the circumference of the distal esophagus (-Fig.4a). The band was removed surgically, leaving an intraoperative trans-

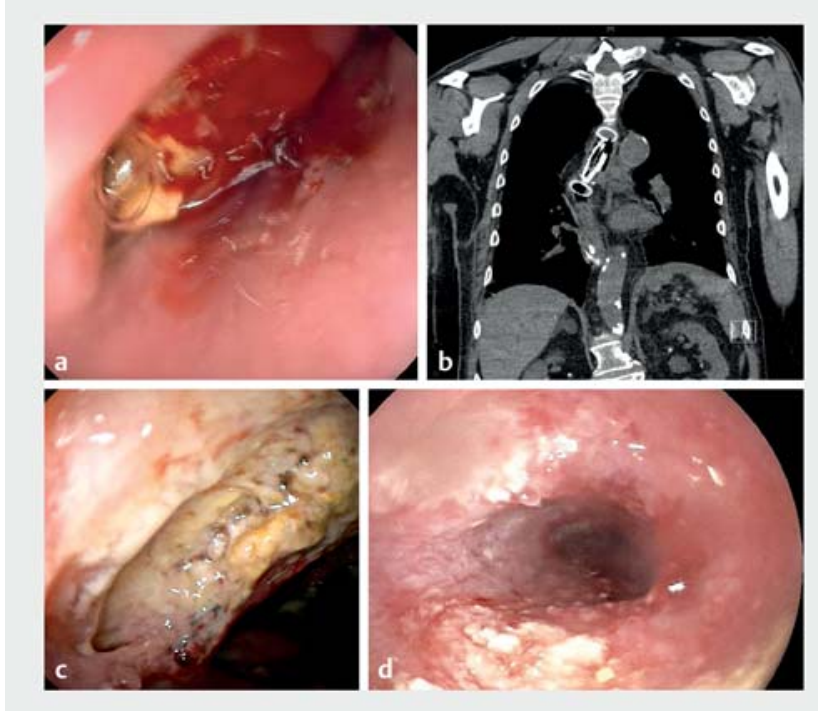

- Fig. 2 a A small abscess at the circular stapler line 14 days after subtotal oesophagectomy with intrathoracic anastomosis. b Thorax CT shows exact location of the VAC Stent and no signs of mediastinitis or abscess formation. c The leak at the staple line cleaned up 5 days later. $\mathbf{d}$ Good granulation tissue is shown with a shallow cavity completely covered by granulation tissue.

mural gap ( $\triangleright$ Fig. 4b) plugged by insertion of a VACStent with a constant negative pressure of $-80 \mathrm{mmHg}$. During the completely unremarkable postoperative course, the patient was able to swallow liquids and also a blended diet. After 4 days, the VACStent was removed, leaving good granulation tissue within a sealed-off gap. On postoperative day 6, an upper gastrointestinal contrast series using swallowed contrast fluid demonstrated a sealed situation with good passage of the gastro-esophageal junction (GEJ). The patient was discharged on postoperative day 8 , and during follow-up, no further dysphagia was reported.

\section{Discussion}

These initial clinical cases demonstrates that the VACStent was easy to insert and able to seal off esophageal leakage and anastomotic failure. The VACStent concept, with its combined benefits of the EVT and covered SEMS, has potential to improve clinical management of these otherwise often challenging cases [3].

The VACStent is a hybrid system comprising a fully covered stent and a polyurethane sponge cylinder connected to a vacuum pump. Clinical experience proves that the sponge cylinder is able to exert circumferential continuous negative pressure on the intestinal wall, which becomes the driving force for both elimination of inflammatory secretions and anchoring of the VACStent. Thus, no migration of any applied VACStent has been observed to date. This is impressive, since the GEJ is a challenging region in which most covered SEMS will dislodge $[2,4]$. In line with the basic concept, use of continuous negative pressure, as measured by the suction pump, resulted in no sig- 

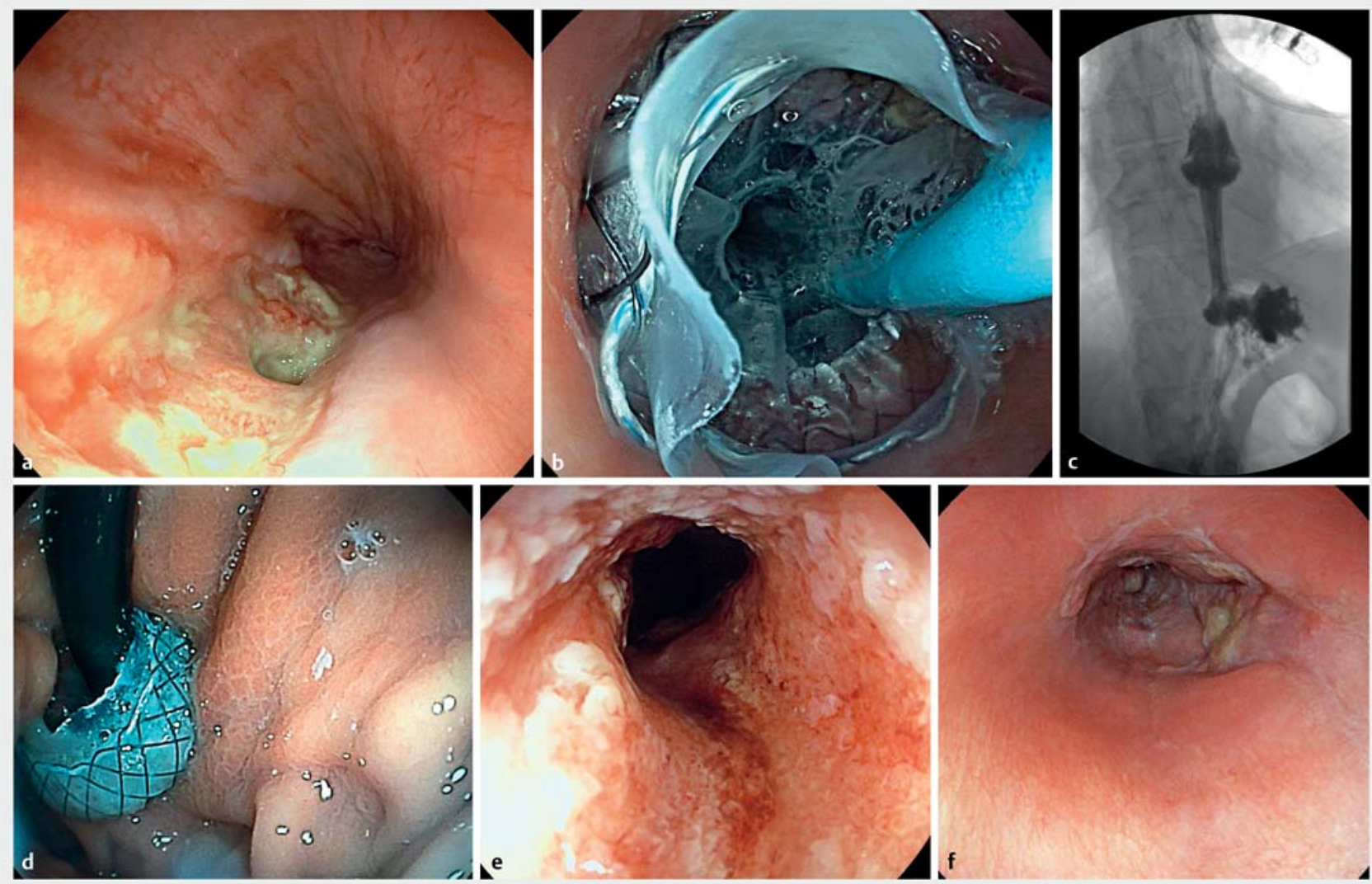

- Fig. 3 a Superficial rather broad mucosal dehiscence from 7.00 to 9.00 with a persisting small transmural gap at the top of the tear. $\mathbf{b}$ The applicated VAC Stent has already expanded, the suction catheter is seen at 2.00. c Gastrografin contrast liquid was swallowed and passes through the VAC Stent into the gastric fundus. $\mathbf{d}$ The endoscope is passed through the VAC Stent and the positioning of the distal bulge of the stent at the esophageal-gastric junction is demonstrated. e The mucosal tear is now completely filled with granion tissue, and no further leak was found. $\mathrm{f}$ One month later no further scar or stenosis was detected.

nificant problems in any of these cases. In other words, no leaks or sponge obstructions were observed in these patients, implying that the EVT principle also works in this hybrid stent system.

The sponge material employed, open-cell polyurethane foam, is identical to that successfully used for years in negative-pressure wound therapy (NPWT) [10] and the endoscopic EVT system (BBraun AG, Melsungen, Germany). Thus, we observed clearing of debris and excellent induction of granulation tissue, reflecting good wound healing. Given this evidence we believe that the VACStent is able to seal off the sponge cylinder against the intestinal lumen, thereby allowing continuous negative pressure to anchor the stent and heal the perforation. Even in case of intermittent leaks between the ends of the covered stent and the intestinal wall, the suction force of the negative pressure reapproximates the flexible intestinal wall and closes the gap. That this indeed does happen in clinical practice is reflected by the good experience documented with the pressure control via the vacuum pump.

The drawback of endoluminal EVT is obstruction of the gastroinstinal tract passage $[4,5]$. This causes microaspiration and mandates parenteral nutrition or application of an additional feeding tube running alongside or through the sponge. Our ex- perience demonstrates that the VACStent offers the option of oral nutrition. Of course, swallowing is hampered somewhat, because the covered stent blocks the active peristaltic wave over the length of the stent and passage is only passive. As evidenced by our clinical observations and radiographic findings, this may slow down passage but still allow it.

Clinical success of this new device will be subject to several parameters, which are and will be addressed by ongoing and future studies $[9,10]$. One major parameter is the selected negative pressure $[7,8,11]$. This level will not only impact stent-migration stability and the rate of wound healing but also determine the extent of ingrowth into the sponge. Due to the cell size and the consistency of the wound surface, the sponge may be suctioned strongly to the wound bed and thus adhere there quite tightly. This ingrowth is the reason why the sponge must be changed every 3 to 7 days $[12,13]$. In other words, the VACStent must be removed at least once a week. Our experience shows that the duration of the needed VACStent treatment depends on the degree of leak dehiscence, possible presence of an abscess or larger extraluminal cavity, and the time between initial diagnosis and sealing of the leakage. In the one case of iatrogenic intraoperative transmural defect, just one 

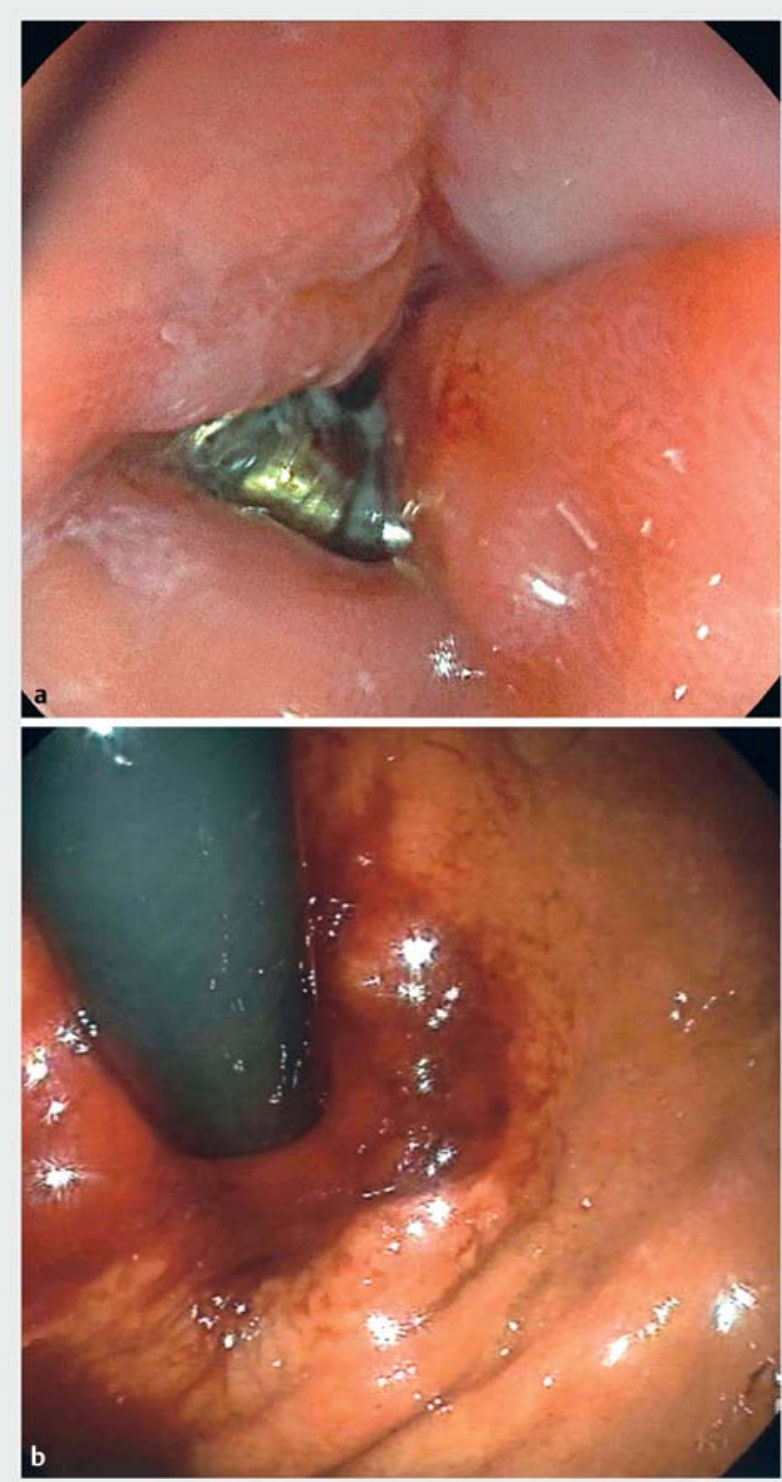

- Fig. 4 a The distal esophagus is invaded by the LINX band with a functional stenosis. More than one-third of the circumference has migrated into the lumen. $\mathbf{b}$ After removal of the LINX band a transmural leak sustained at the location where the LINX band had invaded.

VACStent application for 4 days was sufficient for successful clinical closure. Similarly, successful brief durations of treatment were observed in iatrogenic esophageal perforation managed by endoluminal EVT [14].

There are some limitations of this study, as three cases are not very representative of the different types of upper gastrointestinal defects and none of the patients exhibited a large defect or a greatly infected cavity. Larger mediastinal wound cavities may be treated either directly with a VACStent, when the opening of the cavity is large enough to admit negative pressure within, or as a first step, by intracavitary EVT. The next step may involve a VACStent as in the management of our second case.

\section{Conclusions}

Future trials should address whether the efficacy of the VACStent is comparable to standard EVT in complex situations and how cost-effective it is. If this can be proven, then the VACStent is first choice for early detected defects or anastomotic leaks, but also for complex cavities, if enough drainage capacity is provided by the sponge cylinder to allow NPWT.

Most VACStent indications, however, will be for anastomotic leakage following oncological gastroesophageal surgery or bariatric procedures, e.g. sleeve gastrectomy and gastric bypass. Current studies will reveal the role that this innovative device will play in the armory of interventional endoscopists.

\section{Acknowledgments}

This work was supported by a research fund of the VacStent MedTech AG given to the University Witten/Herdecke.

\section{Competing interests}

Prof. M. Heiss is a consultant for VacStent MedTech AG and has patent ownership interests.

\section{References}

[1] Paspatis GA, Dumonceau JM, Barthet M et al. Diagnosis and management of iatrogenic endoscopic perforations: European Society of Gastrointestinal Endoscopy (ESGE) Position Statement. Endoscopy 2014; 46: 693-711

[2] Rodrigues-Pinto E, Repici A, Donatelli G et al. International multicenter expert survey on endoscopic treatment of upper gastrointestinal anastomotic leaks. Endosc Int Open 2019; 7: E1671-E1682

[3] Chon SH, Bartella I, Bürger M et al. VACStent: a new option for endoscopic vacuum therapy in patients with esophageal anastomotic leaks after upper gastrointestinal surgery. Endoscopy 2020; 52: E166-E167

[4] Rausa E, Asti E, Aiolfi A et al. Comparison of endoscopic vacuum therapy versus endoscopic stenting for esophageal leaks: systematic review and meta-analysis. Dis Esophagus 2018; 31: doi:10.1093/ dote/doy060

[5] Berlth F, Bludau M, Plum PS et al. Self-expanding metal stents versus endoscopic vacuum therapy in anastomotic leak treatment after oncologic gastroesophageal surgery. J Gastrointest Surg 2019; 23: 6775

[6] Wedemeyer ], Schneider A, Manns MP et al. Endoscopic vacuum-assisted closure of upper intestinal anastomotic leaks. Gastrointest Endosc 2008; 67: 708-711

[7] Schorsch T, Müller C, Loske G. Endoscopic vacuum therapy of anastomotic leakage and iatrogenic perforation in the esophagus. Surg Endosc 2013; 27: 2040-2045

[8] Jung CFM, Müller-Dornieden A, Gaedcke J et al. Impact of endoscopic vacuum therapy with low negative pressure for esophageal perforations and postoperative anastomotic esophageal leaks [published online ahead of print, 2020 Feb 11]. Digestion 2020: 1-11. doi:10.1159/000506101

[9] Lange J, Heiss MM. VAC-Stent: Eine prospektive Machbarkeitsstudie zur Behandlung von Leckagen im Gastrointestinaltrakt durch die Kombination von gecovertem Nitinol-Stent und Unterdruckwundbehandlung. Deutsches Register klinischer Studien. DRKS00016048 
[10] Ubbink DT, Westerbos S], Evans D et al. Topical negative pressure for treating chronic wounds. Cochrane Database Syst Rev 2008; 3 : CD001898

[11] Chon SH. Feasibility and efficacy of a combination of a SEMS and vacuum wound treatment (VACStent). ClinicalTrials.gov Identifier: NCT03962179

[12] Morykwas MJ, Argenta LC, Shelton-Brown El et al. Vacuum-assisted closure: a new method for wound control and treatment: animal studies and basic foundation. Ann Plast Surg 1997; 38: 553-562
[13] Schniewind B, Schafmayer C, Both M et al. Ingrowth and device disintegration in an intralobar abscess cavity during endosponge therapy for esophageal anastomotic leakage. Endoscopy 2011; 43: E64-E65

[14] Kuehn F, Loske G, Schiffmann L et al. Endoscopic vacuum therapy for various defects of the upper gastrointestinal tract. Surg Endosc 2017; 31: $3449-3458$ 